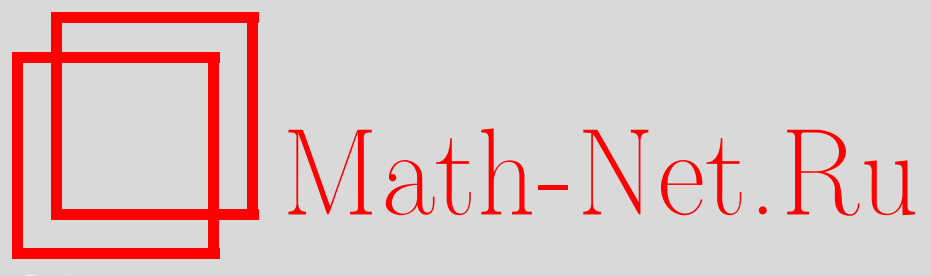

Ю. А. Церковников, Оптимальный способ обрыва цепочки уравнений для двухвременных функций Грина, $T M \Phi, 2006$, том 147, номер 3, 503-510

DOI: https://doi.org/10.4213/tmf1991

Использование Общероссийского математического портала Math-Net.Ru подразумевает, что вы прочитали и согласны с пользовательским соглашением http://www . mathnet.ru/rus/agreement

Параметры загрузки:

IP : 54.174 .149 .18

26 апреля 2023 г., 14:15:37

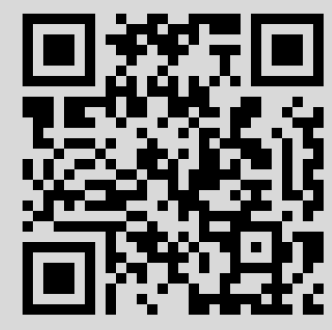




\section{ОПТИМАЛЬНЫЙ СПОСОБ ОБРЫВА ЦЕПОЧКИ УРАВНЕНИЙ ДЛЯ ДВУХВРЕМЕННЫХ ФУНКЦИЙ ГРИНА}

На примере цепочки уравнений для системы бозе-частиц с парным взаимодействием разработана методика расцепления на втором звене цепочки. Получено приближение типа взаимодействующих мод, приводящее к системе нелинейных уравнений для одно-, двух- и трехчастичных функций.

Ключевые слова: функции Грина, цепочки уравнений, приближение взаимодействующих мод.

\section{1. ИСХОДНЫЕ УРАВНЕНИЯ}

Будем исходить из цепочки уравнений для двухвременных температурных функций Грина, рассмотренной в работе [1] и в ряде предыдущих работ. Приведем сначала в общем виде основные соотношения, используемые в работе. Считаем, что последовательность динамических переменных $a(1), a(2), a(3), \ldots$ связана уравнениями движения $(\hbar=1)$ достаточно общего вида:

$$
i a(n)=\omega(n \mid \bar{n}) a(\bar{n})+\nu(n \mid \overline{n+1}) a(\overline{n+1}) .
$$

Черта над парой аргументов $\bar{n}$ означает свертку по квантовому состоянию, соответствующему номеру " $n$ ", а также и по векторным индексам, если $a(n)$ - операторный столбец. Рассматривая для определенности коммутаторные функции, произведем “ортогонализацию” последовательности $a(n), \quad n=1,2,3, \ldots$, положив $A(1) \equiv a(1)$ и

$$
A(n)=a(n)-\sum_{m=1}^{n-1}\left\langle\left[a(n), A^{+}\left(\bar{m}^{\prime}\right)\right]_{-}\right\rangle \eta^{-1}\left(\bar{m}^{\prime} \mid \bar{m}\right) A(m), \quad n>1,
$$

где усреднение производится по ансамблю с гамильтонианом $H$, таким образом, $\langle\ldots\rangle=Q^{-1} \operatorname{Sp}\left\{e^{-\beta H} \ldots\right\}, \beta=\theta^{-1}$. Коммутатор $\eta\left(m \mid m^{\prime}\right)$ в (1.2) задается формулой

$$
\eta\left(m \mid m^{\prime}\right)=\left\langle\left[A(m), A^{+}\left(m^{\prime}\right)\right]\right\rangle=\left\langle\left[a(m), a^{+}\left(m^{\prime}\right)\right]_{-}\right\rangle_{m-1}
$$

* Математический институт им. В. А. Стеклова РАН, Москва, Россия 
Функции Грина, диагональные по номеру $n$,

$$
\begin{aligned}
G_{E}\left(n \mid n^{\prime}\right) & =\left\langle\left\langle A(n) \mid A^{+}\left(n^{\prime}\right)\right\rangle\right\rangle_{n-1, E}=\left\langle\left\langle a(n) \mid a^{+}\left(n^{\prime}\right)\right\rangle\right\rangle_{n-1}= \\
& =-i \int_{0}^{\infty} d t e^{i E t}\left\langle\left[a(n, t), a^{+}\left(n^{\prime}\right)\right]_{-}\right\rangle_{n-1},
\end{aligned}
$$

определяются уравнениями $(n=1,2,3, \ldots)$

$$
\begin{aligned}
& E G_{E}\left(n \mid n^{\prime}\right)=\eta\left(n \mid n^{\prime}\right)+ \\
& \quad+\left\{\left[\left\langle i \dot{A}(n), A^{+}\left(\bar{n}^{\prime}\right)\right]_{-}\right\rangle+\left\langle\left\langle i \dot{A}(n) \mid-i \dot{A}^{+}\left(\bar{n}^{\prime}\right)\right\rangle\right\rangle_{n, E}\right\} \eta^{-1}\left(\bar{n}^{\prime} \mid \bar{n}\right) G_{E}\left(\bar{n} \mid n^{\prime}\right),
\end{aligned}
$$

из которых может быть составлена обобщенная бесконечная цепная дробь.

Входящие в уравнения неприводимые функции определяются рекуррентными соотношениями

$$
\begin{aligned}
& \left\langle\left\langle A \mid B^{+}\right\rangle\right\rangle_{n, E}=\left\langle\left\langle A \mid B^{+}\right\rangle\right\rangle_{n-1, E}^{-} \\
& \quad-\left\langle\left\langle A \mid A^{+}\left(\bar{n}^{\prime}\right)\right\rangle\right\rangle_{n-1, E}\left\langle\left\langle A\left(\bar{n}^{\prime}\right) \mid A^{+}(\bar{n})\right\rangle\right\rangle_{n-1, E}^{-1}\left\langle\left\langle A(\bar{n}) \mid B^{+}\right\rangle\right\rangle_{n-1, E} .
\end{aligned}
$$

Аналогичные соотношения будем иметь и для одновременны́х коммутаторов.

Недиагональные по $n$ функции, если известны диагональные функции $G_{E}\left(n \mid n^{\prime}\right)$, вычисляются по формулам

$$
\begin{gathered}
\left\langle\left\langle A(n) \mid B^{+}\right\rangle\right\rangle_{n-1, E}=G_{E}\left(n \mid \bar{n}^{\prime}\right) \eta^{-1}\left(\bar{n}^{\prime} \mid \bar{n}\right)\left\{\left\langle\left[A(\bar{n}) \mid B^{+}\right]_{-}\right\rangle+\left\langle\left\langle i \dot{A}(\bar{n}) \mid B^{+}\right\rangle\right\rangle_{n, E}\right\}, \\
\left\langle\left\langle A \mid A^{+}\left(n^{\prime}\right)\right\rangle\right\rangle_{n-1}=\left\{\left\langle\left[A, A^{+}\left(\bar{n}^{\prime}\right)\right]_{-}\right\rangle+\left\langle\left\langle A \mid-i \dot{A}^{+}\left(\bar{n}^{\prime}\right)\right\rangle\right\rangle_{n, E}\right\} \eta^{-1}\left(\bar{n}^{\prime} \mid \bar{n}\right) G_{E}\left(\bar{n} \mid n^{\prime}\right) .
\end{gathered}
$$

\section{2. СИСТЕМА БОЗЕ-ЧАСТИЦ С ПАРНЫМ ВЗАИМОДЕЙСТВИЕМ}

Воспользовавшись представленными в предыдущем разделе формулами, рассмотрим систему бозе-частиц с парным взаимодействием и обсудим возможности получения замкнутых уравнений путем обрыва цепочки на втором шаге. Согласно уравнениям цепочки (1.5) при $n=1,2$ и уравнению (1.8) имеем

$$
\begin{gathered}
\left.E G_{q, E}=1+\left\{\left\langle\left[i \dot{a}_{q}, a_{q}^{+}\right)\right]_{-}\right\rangle+\left\langle\left\langle i \dot{a}_{q} \mid-i a_{q}^{+}\right\rangle\right\rangle_{1, E}\right\} G_{q, E}, \\
E G_{q, E}\left(p, k \mid p^{\prime}, k^{\prime}\right)=\eta_{q}\left(p, k \mid p^{\prime}, k^{\prime}\right)+\left\{\left\langle\left[i \dot{A}_{q}(p, k), A_{q}^{+}\left(\bar{p}^{\prime}, \bar{k}^{\prime}\right)\right]_{-}\right\rangle+\right. \\
\left.+\left\langle\left\langle i \dot{A}_{q}(p, k) \mid-i \dot{A}_{q}^{+}\left(\bar{p}^{\prime}, \bar{k}^{\prime}\right)\right\rangle\right\rangle_{2, E}\right\} \eta_{q}^{-1}\left(\bar{p}^{\prime}, \bar{k}^{\prime} \mid \bar{p}, \bar{k}\right) G_{q, E}\left(\bar{p}, \bar{k} \mid p^{\prime}, k^{\prime}\right), \\
\left\langle\left\langle A_{q}\left(p, k \mid a_{q}^{+}\right)\right\rangle\right\rangle_{E}=\frac{1}{V} \sum_{p_{1}, k_{1}} \nu\left(k_{1}\right) G_{q, E}\left(p, k \mid p_{1}, k_{1}\right) G_{q, E},
\end{gathered}
$$

где

$$
\begin{gathered}
A_{q}(p, k)=a_{p}^{+} a_{p+k} a_{-k+q}-\left\langle\left[a_{p}^{+} a_{p+k} a_{-k+q}, a_{q}^{+}\right]_{-}\right\rangle a_{q} \\
G_{E}\left(1 \mid 1^{\prime}\right) \equiv G_{q, E}=\left\langle\left\langle a_{q} \mid a_{q}^{+}\right\rangle\right\rangle_{E} \\
G_{E}\left(2 \mid 2^{\prime}\right) \equiv G_{q, E}\left(p, k \mid p^{\prime}, k^{\prime}\right)=\left\langle\left\langle a_{p}^{+} a_{p+k} a_{-k+q} \mid a_{-k^{\prime}+q}^{+} a_{p^{\prime}+k^{\prime}}^{+} a_{p^{\prime}}\right\rangle\right\rangle_{1, E}
\end{gathered}
$$


$\eta\left(1 \mid 1^{\prime}\right) \equiv\left\langle\left[a_{p}, a_{q}^{+}\right]_{-}\right\rangle=1$ и $\nu(k)$ - фурье-образ потенциала парного взаимодействия. $\mathrm{B}(2.3)$ в силу условия "ортогональности" $\left\langle\left[A_{q}(p, k), a_{q}^{+}\right]_{-}\right\rangle=0$ не вошел первый член в (1.8) при $n=1$.

Раскроем входящие в $(2.2)$ одновременны́е $(t=0)$ коммутаторы $\eta_{q}\left(p, k \mid p^{\prime}, k^{\prime}\right)$ и $\left\langle\left[i \dot{A}_{q}(p, k), A_{q}^{+}\left(p^{\prime}, k^{\prime}\right)\right]_{-}\right\rangle$, выделив из них все возможные одночастичные спаривания $\left\langle a_{k}^{+}, a_{k}\right\rangle$. Предварительно по аналогии с квантовой теорией поля [2] введем нормальные произведения, положив, например,

$$
\begin{gathered}
a_{p}^{+} a_{p}=\left\langle a_{p}^{+} a_{p}\right\rangle+: a_{p}^{+} a_{p}: \\
a_{q}^{+}: a_{p}^{+} a_{p+k} a_{-k+q}:=\left(\delta_{k, 0}+\delta_{k,-p+q}\right)\left\langle a_{q}^{+} a_{q}\right\rangle: a_{p}^{+} a_{p}:+: a_{q}^{+} a_{p}^{+} a_{p+k} a_{-k+q}: \\
: a_{p}^{+} a_{p+k} a_{-k+q}: a_{q}^{+}=\left(\delta_{k, 0}+\delta_{k,-p+q}\right)\left\langle a_{q} a_{q}^{+}\right\rangle: a_{p}^{+} a_{p}:+: a_{p}^{+} a_{p+k} a_{-k+q} a_{q}^{+}:
\end{gathered}
$$

Соотношения (2.4)-(2.6) являются тождествами и могут служить для определения нормальных произведений, выделенных точками. Очевидно, что нормальные произведения не зависят от порядка сомножителей, так что все операторы рождения $a^{+}$могут быть перемещены влево от $a$, и

$$
: a_{p}^{+} a_{p+k} a_{-k+q} a_{q}^{+}:=: a_{q}^{+} a_{p}^{+} a_{p+k} a_{-k+q}:
$$

Исходя из определения неприводимой функции при $n=1$, производя спаривания коммутирующих операторов, с учетом (2.4)-(2.7) получаем

$$
\begin{aligned}
& \eta_{q}\left(p, k \mid p^{\prime}, k^{\prime}\right)=\left\langle\left[a_{p}^{+} a_{p+k} a_{-k+q}, a_{-k^{\prime}+q}^{+} a_{p^{\prime}+k^{\prime}}^{+} a_{p^{\prime}}\right]_{-}\right\rangle_{1}= \\
& =\langle[ \\
& \left.\left.\quad \times a_{p}^{+} a_{p+k} a_{-k+q}:,: a_{-k^{\prime}+q}^{+} a_{p^{\prime}+k^{\prime}}^{+} a_{p^{\prime}}:\right]_{-}\right\rangle=\left\{n_{p}\left(1+n_{p+k}+n_{-k+q}\right)-n_{p+k} n_{-k+q}\right\} \times \\
& \quad \times \delta_{p, p^{\prime}}\left(\delta_{k, k^{\prime}}+\delta_{-k-p+q, k}\right)+\left\langle: a_{p}^{+} a_{p^{\prime}+k}^{+} a_{p^{\prime}} a_{p+k}:\right\rangle\left(\delta_{k, k^{\prime}}+\delta_{-k+q, p^{\prime}+k^{\prime}}\right)+ \\
& \quad+\left\langle: a_{p}^{+} a_{p^{\prime}-p-k+q}^{+} a_{p^{\prime}} a_{-k+q}:\right\rangle\left(\delta_{-k-p+q, k^{\prime}}+\delta_{p+k, p^{\prime}+k^{\prime}}\right)- \\
& \quad-\left\langle: a_{p+k^{\prime}}^{+} a_{-k^{\prime}+q}^{+} a_{-k+q} a_{p+k}:\right\rangle \delta_{p, p^{\prime}}=\eta_{q}(p, k) 2 \delta_{q}\left(p, k \mid p^{\prime}, k^{\prime}\right)+u_{q}\left(p, k \mid p^{\prime}, k^{\prime}\right) .
\end{aligned}
$$

В правой части (2.8)

$$
\delta_{q}\left(p, k \mid p^{\prime}, k^{\prime}\right)=\frac{1}{2} \delta_{p, p^{\prime}}\left(\delta_{k, k^{\prime}}+\delta_{-k-p+q, k}\right)
$$

- обобщенный символ Кронекера; определения величин $\eta_{q}(p, k)$ и $u_{q}\left(p, k \mid p^{\prime}, k^{\prime}\right)$ следуют из двух последних равенств в (2.8). Обратная матрица $\eta_{q}^{-1}\left(p, k \mid p^{\prime}, k^{\prime}\right)$ может быть получена из интегрального уравнения с неоднородностью, пропорциональной $\eta_{q}^{-1}(p, k)$.

Аналогично (2.8) преобразуется и входящий $\quad$ в $\quad(2.2)$ коммутатор $\left\langle\left[i \dot{A}_{q}(p, k), A_{q}^{+}\left(p^{\prime}, k^{\prime}\right)\right]_{-}\right\rangle$. Не приводя подробных выкладок, представим только конечный результат вычислений:

$$
\begin{aligned}
& \left\langle\left[i \dot{A}_{q}(p, k), A_{q}^{+}\left(p^{\prime}, k^{\prime}\right)\right]_{-}\right\rangle=\varepsilon_{q}(p, k) \eta_{q}\left(p, k \mid p^{\prime}, k^{\prime}\right)+ \\
& +V_{q}(p, k \mid \bar{p}, \bar{k}) \eta_{q}\left(\bar{p}, \bar{k} \mid p^{\prime}, k^{\prime}\right)+U_{q}\left(p, k \mid p^{\prime}, k^{\prime}\right),
\end{aligned}
$$

где

$$
\varepsilon_{q}(p, k)=\varepsilon_{-k+q}+\varepsilon_{p+k}-\varepsilon_{p}, \quad \varepsilon_{q}=\frac{q^{2}}{2 m}-\mu+\frac{N}{V} \nu(0)+\frac{1}{V} \sum_{k_{1}} \nu\left(k_{1}\right) n_{-k_{1}+q},
$$




$$
\begin{aligned}
V_{q}\left(p, k \mid p^{\prime}, k^{\prime}\right)=\frac{1}{V} & \left\{\left(1+n_{p+k}+n_{-k+q}\right) \frac{1}{2}\left(\nu\left(k-k^{\prime}\right)+\nu\left(k+k^{\prime}+p-q\right)\right) \delta_{p, p^{\prime}}+\right. \\
& +\left(n_{p}-n_{p+k}\right)\left(\nu(k)+\nu\left(p-p^{\prime}\right)\right) \delta_{k, k^{\prime}}+ \\
& \left.+\left(n_{p}-n_{-k+q}\right)\left(\nu(p+k-q)+\nu\left(p-p^{\prime}\right)\right) \delta_{-k-p+q, k^{\prime}}\right\}
\end{aligned}
$$

получены в результате всех допустимых спариваний в $i \dot{A}_{q}(p, k)$. В результате выделения нормальных произведений и спариваний по разные стороны от запятой, используя уравнения движения

$$
\begin{gathered}
i \dot{a}_{p}=\left\{\frac{p^{2}}{2 m}-\mu+\frac{N}{V} \nu(0)+\frac{1}{V} \sum_{k_{1}} \nu\left(k_{1}\right) n_{-k_{1}+q}\right\} a_{q}+i \dot{a}_{q}^{i r}, \\
i \dot{a}_{q}^{i r}=\frac{1}{V} \sum_{p_{1} k_{1}} \nu\left(k_{1}\right): a_{p_{1}}^{+} a_{p_{1}+k_{1}} a_{-k_{1}+q}:
\end{gathered}
$$

где $i \dot{a}_{q}^{i r}-$ не приводимая к $a_{q}$ часть оператора $i \dot{a}_{q}$, получаем, что $U_{q}=U_{q}^{(1)}+U_{q}^{(2)}+$ $U_{q}^{(3)}+U_{q}^{(4)}$, где

$$
\begin{gathered}
U_{q}^{(1)}\left(p, k \mid p^{\prime}, k^{\prime}\right)=U_{q}^{(1)}(p, k) \delta_{p, p^{\prime}}\left(\delta_{k, k^{\prime}}+\delta_{-k-p+q, k}\right), \\
U_{q}^{(1)}(p, k)=\left(n_{p}-n_{p+k}\right)\left\langle: i \dot{a}_{-k+q}^{i r} a_{-k+q}^{+}:\right\rangle+\left(n_{p}-n_{-k+q}\right)\left\langle: i \dot{a}_{p+k}^{i r} a_{p+k}^{+}:\right\rangle+ \\
+\left(1+n_{p+k}+n_{-k+q}\right)\left\langle: i \dot{a}_{p}^{i r} a_{p}:\right\rangle \\
U_{q}^{(2)}\left(p, k \mid p^{\prime}, k^{\prime}\right)=\delta_{p, p^{\prime}}\left(n_{p}-n_{-k^{\prime}+q}\right)\left\langle:\left(a_{p+k}\left[i \dot{a}_{-k+q}^{i r}, a_{-k^{\prime}+q}^{+}\right]_{-}+\right.\right. \\
\left.\left.+\left[i \dot{a}_{p+k}^{i r}, a_{-k^{\prime}+q}^{+}\right]_{-} a_{-k+q}\right) a_{p+k^{\prime}}^{+}:\right\rangle+\left(n_{p^{\prime}}-n_{-k^{\prime}+q}\right) \times \\
\times\left\langle:\left(a_{p}^{+} a_{p+k}\left[a_{p^{\prime}},\left[i \dot{a}_{-k+q}^{i r}, a_{-k^{\prime}+q}^{+}\right]_{-}\right]_{-}+a_{p}^{+}\left[a_{p^{\prime}},\left[i \dot{a}_{p+k}^{i r}, a_{-k^{\prime}+q}^{+}\right]_{-}\right]_{-} a_{-k+q}+\right.\right. \\
\left.\left.+\left[a_{p^{\prime}},\left[i \dot{a}_{p}^{+i r}, a_{-k^{\prime}+q}^{+}\right]_{-}\right]_{-} a_{p+k} a_{-k+q}\right) a_{p^{\prime}+k^{\prime}}^{+}:\right\rangle+\left(k^{\prime} \rightarrow-k^{\prime}-p^{\prime}+q\right),
\end{gathered}
$$

$$
\begin{aligned}
U_{q}^{(3)}\left(p, k \mid p^{\prime}, k^{\prime}\right)=(1 & \left.+n_{p^{\prime}+k^{\prime}}+n_{-k^{\prime}+q}\right) \times \\
& \left.\times\left\langle: a_{p}^{+} a_{p+k}\left[\left[i \dot{a}_{-k+q}^{i r}, a_{-k^{\prime}+q}^{+}\right]_{-}\right]_{-}, a_{p^{\prime}+k^{\prime}}^{+}\right]_{-} a_{p^{\prime}}:\right\rangle+ \\
& +\left(\delta_{k, k^{\prime}}+\delta_{-k+q, p^{\prime}+k^{\prime}}\right)\left\{\left(1+n_{-k+q}+n_{p^{\prime}+k}\right) \times\right. \\
& \times\left\langle:\left(a_{p}^{+}\left[i \dot{a}_{p+k}^{i r}, a_{p^{\prime}+k}^{+}\right]_{-}+\left[i \dot{a}_{p}^{+i r}, a_{p^{\prime}+k}^{+}\right]_{-} a_{p+k}\right) a_{p^{\prime}}:\right\rangle+ \\
& \left.+\left(n_{p^{\prime}}-n_{-k+q}\right)\left\langle:\left(a_{p}^{+}\left[a_{p^{\prime}}, i \dot{a}_{p+k}^{i r}\right]_{-}+\left[a_{p^{\prime}}, i \dot{a}_{p}^{+i r}\right]_{-} a_{p+k}\right) a_{p^{\prime}+k}^{+}:\right\rangle\right\}+ \\
& +(k \rightarrow-k-p+q),
\end{aligned}
$$

$$
\begin{aligned}
& U_{q}^{(4)}\left(p, k \mid p^{\prime}, k^{\prime}\right)=\left\{\left\langle:\left(a_{p}^{+} a_{p+k}\left[i \dot{a}_{-k+q}^{i r}, a_{-k^{\prime}+q}^{+}\right]_{-}+a_{p}^{+}\left[i \dot{a}_{p+k}^{i r}, a_{-k^{\prime}+q}^{+}\right]_{-} a_{-k+q}+\right.\right.\right. \\
& \left.\left.\left.+\left[i \dot{a}_{p}^{+i r}, a_{-k^{\prime}+q}^{+}\right]_{-} a_{p+k} a_{-k+q}\right) a_{p^{\prime}+k^{\prime}}^{+} a_{p^{\prime}}:\right\rangle+\left(k^{\prime} \rightarrow-k^{\prime}-p^{\prime}+q\right)\right\}+ \\
& \quad+\left\{\left\langle:\left(a_{p}^{+} i \dot{a}_{p+k}^{i r}+i \dot{a}_{p}^{+i r} a_{p+k}\right) a_{p^{\prime}+k}^{+} a_{p^{\prime}}:\right\rangle-\left\langle: a_{p+k} i \dot{a}_{-k+q}^{i r} a_{-k^{\prime}+q}^{+} a_{p+k^{\prime}}^{+}\right\rangle\right\rangle-
\end{aligned}
$$




$$
\begin{aligned}
& \left.-\left\langle: a_{p}^{+} a_{p+k}\left[a_{p^{\prime}}, i \dot{a}_{-k+q}^{i r}\right]_{-} a_{k^{\prime}+q}^{+} a_{p^{\prime}+k^{\prime}}:\right\rangle+(k \rightarrow-k-p+q)\right\}- \\
& -\left\langle:\left[a_{p^{\prime}}, i \dot{a}_{p}^{+i r}\right]_{-} a_{p+k} a_{-k+q} a_{-k^{\prime}+q}^{+} a_{p^{\prime}+k^{\prime}}^{+}:\right\rangle .
\end{aligned}
$$

В (2.15)-(2.17) для краткости не выписаны явно члены, получающиеся заменами $k \rightarrow-k-p+q$ и $k^{\prime} \rightarrow-k^{\prime}-p^{\prime}+q^{\prime}$.

С учетом обозначений, введенных в $(2.11),(2.12),(2.14)-(2.17)$, представим второе уравнение цепочки (2.2) в виде

$$
\begin{aligned}
E G_{q, E}\left(p, k \mid p^{\prime}, k^{\prime}\right)=\eta_{q} & \left(p, k \mid p^{\prime}, k^{\prime}\right)+\varepsilon_{q}(p, k) G_{q, E}\left(p, k \mid p^{\prime}, k^{\prime}\right)+ \\
& +V_{q}(p, k \mid \bar{p}, \bar{k}) G_{q, E}\left(\bar{p}, \bar{k} \mid p^{\prime}, k^{\prime}\right)+ \\
& +\left\{U_{q}\left(p, k \mid \bar{p}^{\prime}, \bar{k}^{\prime}\right)+\left\langle\left\langle i \dot{A}_{q}(p, k) \mid-i A_{q}^{+}\left(\bar{p}^{\prime}, \bar{k}^{\prime}\right)\right\rangle\right\rangle_{2, E}\right\} \times \\
& \times \eta_{q}^{-1}\left(\bar{p}^{\prime}, \bar{k}^{\prime} \mid \bar{p}, \bar{k}\right) G_{q, E}\left(\bar{p}, \bar{k} \mid p^{\prime}, k^{\prime}\right) .
\end{aligned}
$$

В этом уравнении легко устанавливаются порядки членов по взаимодействию $\nu(k)$. Так, величины $\eta_{q}(p, k)$, введенные в $(2.8)$, и $\varepsilon_{q}(p, k)$ из $(2.11)$ имеют нулевой порядок, коэффициент $V_{q}\left(p, k \mid p^{\prime}, k^{\prime}\right)(2.12)$ линеен по $\nu$, а величины $U_{q}\left(p, k \mid p^{\prime}, k^{\prime}\right)$ квадратичны (имеют порядок $\nu^{2}$ ).

Действительно, нормальные произведения в $U_{q}\left(p, k \mid p^{\prime}, k^{\prime}\right)$ такие, например, как $\left\langle: a_{q}^{+} a_{p}^{+} a_{p+k} a_{-k+q}:\right\rangle, \quad\left\langle: a_{p}^{+} a_{p_{1}}^{+} a_{p_{1}+k_{1}} a_{-k_{1}-k+q} a_{p^{\prime}-p-k+q}^{+} a_{p^{\prime}}:\right\rangle$, при $\nu=0$ обращаются в нуль по определению. В этом можно убедиться и непосредственно, воспользовавшись спектральным представлением [3] формулы (2.3):

$$
\begin{aligned}
& \left\langle: a_{q}^{+} a_{p}^{+} a_{p+k} a_{-k+q}:\right\rangle=\frac{1}{2 \pi} \int_{-\infty}^{\infty} \frac{d \omega}{e^{\beta \omega}-1} \times \\
& \quad \times \frac{1}{V} \sum \nu\left(k_{1}\right)\left\{G_{q, \omega-i \varepsilon}\left(p, k \mid p_{1}, k_{1}\right) G_{q, \omega-i \varepsilon}-G_{q, \omega+i \varepsilon}\left(p, k \mid p_{1}, k_{1}\right) G_{q, \omega+i \varepsilon}\right\},
\end{aligned}
$$

где в правую часть $(2.19)$ входит $\nu\left(k_{1}\right)$. Кроме того, $\nu(k)$ входит и в комбинации типа $\left\langle: i \dot{a}_{-k+q}^{i r} a_{-k+q}^{+}:\right\rangle$явно. В результате получаем, что $U_{q}\left(p, k \mid p^{\prime}, k^{\prime}\right) \sim \nu^{2}$.

Наконец, последний член в фигурных скобках (2.18), зависящий от $E$, имеет очевидный порядок $\nu^{2}$.

Полагая $\eta_{q}\left(p, k \mid p^{\prime}, k^{\prime}\right) \cong \eta_{q}(p, k) 2 \delta_{q}\left(p, k \mid p^{\prime}, k^{\prime}\right)$ в $(2.8)$, сохраняя $V_{q}\left(p, k \mid p^{\prime}, k^{\prime}\right)$ (см. (2.12)) и отбрасывая все остальные (порядка $\nu^{2}$ ) члены в правой части (2.18), приходим к линейному интегральному уравнению

$$
\begin{aligned}
G_{q, E}\left(p, k \mid p^{\prime}, k^{\prime}\right)=\left(E-\varepsilon_{q}(p, k)\right)^{-1} \eta_{q}(p, k) \delta_{p, p^{\prime}}\left(\delta_{k, k^{\prime}}+\delta_{-k-p+q, k}\right)+ \\
\quad+\left(E-\varepsilon_{q}(p, k)\right)^{-1} V_{q}(p, k \mid \bar{p}, \bar{k}) G_{q, E}\left(\bar{p}, \bar{k} \mid p^{\prime}, k^{\prime}\right)= \\
=\left(E-\varepsilon_{q}(p, k)\right)^{-1} 2 \delta_{q}\left(p, k \mid p^{\prime}, k^{\prime}\right)+ \\
\quad+\left(E-\varepsilon_{q}(p, k)\right)^{-1} \Theta_{q, E}\left(p, k \mid p^{\prime}, k^{\prime}\right)\left(E-\varepsilon_{q}\left(p^{\prime}, k^{\prime}\right)\right)^{-1} \eta_{q}\left(p^{\prime}, k^{\prime}\right),
\end{aligned}
$$

где $\Theta_{q, E}\left(p, k \mid p^{\prime}, k^{\prime}\right)$ - решение уравнения Липпмана-Швингера,

$$
\Theta_{q, E}\left(p, k \mid p^{\prime}, k^{\prime}\right)=V_{q}\left(p, k \mid p^{\prime}, k^{\prime}\right)+V_{q}(p, k \mid \bar{p}, \bar{k})\left(E-\varepsilon_{q}(\bar{p}, \bar{k})\right)^{-1} \Theta_{q, E}\left(\bar{p}, \bar{k} \mid p^{\prime}, k^{\prime}\right) .
$$


Уравнение (2.21), как известно, может быть использовано для перенормировки двухчастичного взаимодействия или экранировки дальнодействующих сил (см., например, [4]). Целью настоящей работы является более детальный учет взаимодействия, включающий члены порядка $\nu^{2}$, входящие не только в стационарные величины $U_{q}\left(p, k \mid p^{\prime}, k^{\prime}\right)$, но и в $\left\langle\left\langle i \dot{A}_{q}(p, k) \mid-i \dot{A}_{q}^{+}\left(p^{\prime}, k^{\prime}\right)\right\rangle\right\rangle_{2, E}$ в уравнении $(2.18)$.

\section{3. ОПТИМАЛЬНОЕ РАСЦЕПЛЕНИЕ ЦЕПОЧКИ УРАВНЕНИЙ}

Полученные выше соотношения (2.1), (2.2) (или (2.18)) и (2.3) представляют собой по сути своей тождества и превращаются в уравнения, если произвести конкретное расцепление в (2.18). Предварительно введем вспомогательную функцию $\widetilde{G}_{q, E}\left(p, k \mid p^{\prime}, k^{\prime}\right)$, которая позволит учесть величины порядка $\nu^{2}$ в обоих членах, расположенных в фигурных скобках в (2.18). Функция $\widetilde{G}_{q, E}\left(p, k \mid p^{\prime}, k^{\prime}\right)$ получается из $G_{q, E}\left(p, k \mid p^{\prime}, k^{\prime}\right)=\left\langle\left\langle a_{p}^{+} a_{p+k} a_{-k+q} \mid a_{-k^{\prime}+q}^{+} a_{p^{\prime}+k^{\prime}}^{+} a_{p^{\prime}}\right\rangle\right\rangle_{1, E}$ спариванием операторов по разные стороны от вертикальной черты. В результате получаем

$$
\begin{gathered}
\widetilde{G}_{q, E}\left(p, k \mid p^{\prime}, k^{\prime}\right)=\left\langle\left\langle a_{p}^{+} a_{p+k} a_{-k+q} \mid a_{-k+q}^{+} a_{p+k}^{+} a_{p}\right\rangle\right\rangle_{E} \delta_{p, p^{\prime}}\left(\delta_{k, k^{\prime}}+\delta_{-k-p+q, k}\right)= \\
=G_{q, E}(p, k) 2 \delta_{q}\left(p, k \mid p^{\prime}, k^{\prime}\right)= \\
=-i \int_{0}^{\infty} d t e^{i E t}\left\{\left\langle a_{p}^{+}(t) a_{p}^{+}\right\rangle\left\langle a_{p+k}(t) a_{p+k}^{+}\right\rangle\left\langle a_{-k+q}(t) a_{-k+q}^{+}\right\rangle-\right. \\
\left.\quad-\left\langle a_{p} a_{p}^{+}(t)\right\rangle\left\langle a_{p+k}^{+} a_{p+k}(t)\right\rangle\left\langle a_{-k+q}^{+} a_{-k+q}(t)\right\rangle\right\} 2 \delta_{q}\left(p, k \mid p^{\prime}, k^{\prime}\right),
\end{gathered}
$$

где нижними скобками отмечены необходимые спаривания, а явный вид операции представлен последним равенством в (3.1).

Аналогично получаем и уравнение для функции (3.1):

$$
\begin{aligned}
E \widetilde{G}_{q, E}(p, k)= & \eta_{q}(p, k)+\left\langle\left\langle[\left.\underbrace{+}_{p}\right|_{p+k} a_{-k+q}, H]_{-} \mid a_{-k+q}^{+} a_{p+k}^{+} a_{p}\right\rangle\right\rangle_{E}= \\
= & \eta_{q}(p, k)-i \int_{0}^{\infty} d t e^{i E t}\left\{\left\langle i \dot{a}_{p}^{+}(t) a_{p}\right\rangle\left\langle a_{p+k}(t) a_{p+k}^{+}\right\rangle\left\langle a_{-k+q}(t) a_{-k+q}^{+}\right\rangle+\right. \\
& +\left\langle a_{p}^{+}(t) a_{p}\right\rangle\left\langle i \dot{a}_{p+k}(t) a_{p+k}^{+}\right\rangle\left\langle a_{-k+q}(t) a_{-k+q}^{+}\right\rangle+ \\
& +\left\langle a_{p}^{+}(t) a_{p}\right\rangle\left\langle a_{p+k}(t) a_{p+k}^{+}\right\rangle\left\langle i \dot{a}_{-k+q}(t) a_{-k+q}^{+}\right\rangle- \\
& \left.- \text {члены с переставленными } a \text { и } a^{+}\right\} .
\end{aligned}
$$

Используя обозначения, введенные в (3.1) и $(3.2)$, и умножая на $\widetilde{G}_{q}^{-1}(p, k) \widetilde{G}_{q}(p, k)$ второй член в правой части (3.2), последовательно получаем

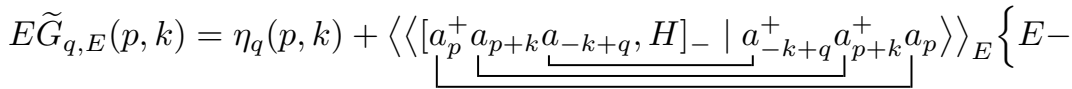

$$
\begin{aligned}
& \left.-\widetilde{G}_{q, E}(p, k)\left\langle\left\langle a_{p}^{+} a_{p+k} a_{-k+q} \mid\left[H, a_{-k+q}^{+} a_{p+k}^{+} a_{p}\right]_{-}\right\rangle\right\rangle_{E}\right\} \eta_{q}^{-1}(p, k) \widetilde{G}_{q, E}(p, k)= \\
& =\eta_{q}(p, k)+\left\{\left\langle\left[a_{p}^{+} \underline{L}_{p+k} a_{-k+q}, H\right]_{-}, a_{-k+q}^{+} a_{p+k}^{+} a_{p}\right]_{-}\right\rangle+ \\
& +\left\langle\left\langle\left[a_{p}^{+} \underline{L}_{p+k}^{\left.a_{-k+q}, H\right]_{-} \mid\left[H, a_{-k+q}^{+}\right.} a_{p+k}^{+} a_{p}\right]_{-}\right\rangle\right\rangle_{E}-
\end{aligned}
$$




$$
\begin{aligned}
& -\left\langle\left\langle[a_{p}^{+} \underbrace{a_{p+k+q}}_{\llcorner+k} a_{-H}]_{-} \mid a_{-k+q}^{+} a_{p+k}^{+} a_{p}\right\rangle\right\rangle \widetilde{G}_{q}^{-1}(p, k) \times \\
& \left.\left.\times\left\langle\langle a_{p}^{+} \underbrace{a_{p+k+q} \mid\left[H, a_{-k+q}^{+}\right.}_{p+k} a_{p+k}^{+} a_{p}]_{-}\right\rangle\right\rangle_{E}\right\} \eta_{q}^{-1}(p, k) \widetilde{G}_{q, E}(p, k)= \\
& \left.=\eta_{q}(p, k)+\varepsilon_{q}(p, k)\right) \widetilde{G}_{q}(p, k)+\left\{U_{q}^{(1)}(p, k)+\right.
\end{aligned}
$$

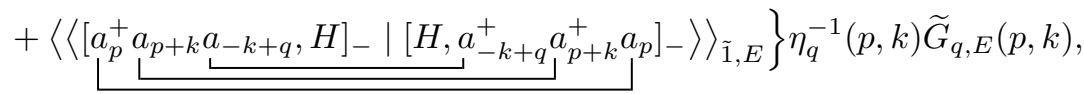

где $U_{q}^{(1)}(p, k)$ определено в $(2.14)$, а $\varepsilon_{q}(p, k)$ - в (2.11).

Уравнение (3.3) по форме близко к (2.18), однако никакой дополнительной информации по сравнению с одночастичной функцией (2.1) оно не содержит, представляя собой тождество для произведения трех одночастичных корреляционных функций.

Используем теперь вспомогательное уравнение, записанное в виде последнего равенства в (3.3). Вычтем из равенства (2.18) равенство (3.3), умноженное на $\widetilde{G}_{q}^{-1}(p, k) G_{q, E}\left(p, k \mid p^{\prime}, k^{\prime}\right)$. В результате член $\left(E-\varepsilon_{q}(p, k)\right) G_{q, E}\left(p, k \mid p^{\prime}, k^{\prime}\right)$ сократится, и мы получим

$$
\begin{aligned}
& \eta_{q}(p, k) \widetilde{G}_{q}^{-1}(p, k) G_{q E}\left(p, k \mid p^{\prime}, k^{\prime}\right)=\eta_{q}\left(p, k \mid p^{\prime}, k^{\prime}\right)+V_{q}(p, k \mid \bar{p}, \bar{k}) G_{q E}\left(\bar{p}, \bar{k} \mid p^{\prime}, k^{\prime}\right)+ \\
&+\left\{U_{q}\left(p, k \mid \bar{p}^{\prime}, \bar{k}^{\prime}\right)+\left\langle\left\langle\left[a_{p}^{+} a_{p+k} a_{-k+q}, H\right]_{-} \mid\left[H, a_{-\bar{k}^{\prime}+q}^{+} a_{\bar{p}^{\prime}+\bar{k}^{\prime}}^{+} a_{\bar{p}^{\prime}}\right]_{-}\right\rangle\right\rangle_{2, E}\right\} \times \\
& \\
& \times \eta_{q}^{-1}\left(\bar{p}^{\prime}, \bar{k}^{\prime} \mid \bar{p}, \bar{k}\right) G_{q E}\left(\bar{p}, \bar{k} \mid p^{\prime}, k^{\prime}\right)- \\
&-\left\{U_{q}^{(1)}(p, k)+\left\langle\left\langle\left[a_{p}^{+} a_{p+k} a_{-k+q}, H\right]_{-} \mid\left[H, a_{-k+q}^{+} a_{p+k}^{+} a_{p}\right]_{-}\right\rangle\right\rangle_{\tilde{1}, E}\right\} \times \\
& \times \eta_{q}^{-1}(p, k) G_{q E}\left(p, k \mid p^{\prime}, k^{\prime}\right) .
\end{aligned}
$$

Произойдет также вычитание из $U_{q}\left(p, k \mid p^{\prime}, k^{\prime}\right)$ величины $U_{q}^{(1)}(p, k)$, в результате чего величина $U_{q}^{(1)}\left(p, k \mid p^{\prime}, k^{\prime}\right)$, определяемая формулой $(2.14)$, заменится на величину $U_{q}^{(1)}(p, k) \eta_{q}^{-1}(p, k) u_{q}\left(p, k \mid p^{\prime}, k^{\prime}\right)$ (см. (2.8)) порядка $\nu^{3}$.

Наконец, в последних (зависящих от $E$ ) членах в разности

$$
\begin{aligned}
& \left\langle\left\langle\left[a_{p}^{+} a_{p+k} a_{-k+q}, H\right]_{-} \mid\left[H, a_{-k^{\prime}+q}^{+} a_{p^{\prime}+k^{\prime}}^{+} a_{p^{\prime}}\right]_{-}\right\rangle\right\rangle_{2, E}- \\
& \quad-\left\langle\left\langle\left[ a_{p}^{+} a_{p+k}^{\left.\left.a_{L+k+q}, H\right]_{-}\left|\left[H, a_{-k+q}^{+} a_{p+k}^{+} a_{p}\right]_{-}\right\rangle\right\rangle_{\tilde{1}, E} \eta_{q}^{-1}(p, k) \eta\left(p, k \mid p^{\prime}, k^{\prime}\right) \sim \nu^{2}}\right.\right.\right.
\end{aligned}
$$

будем учитывать только величины, составленные из выражений типа $\left\langle a_{p}^{+}(t) a_{p}\right\rangle \times$ $\left\langle a_{p+k}(t) a_{p+k}^{+}\right\rangle\left\langle: i \dot{a}_{-k+q}^{i r}(t)\left(-i \dot{a}_{-k+q}^{+i r}\right):\right\rangle$, имеющие порядок $\nu^{2}$. Остальные члены типа $\left.\left\langle a_{p}^{+}(t) a_{p}\right\rangle\left\langle: i \dot{a}_{p+k}^{i r}(t) a_{p+k}^{+}:\right\rangle\left\langle: a_{-k+q}(t) i \dot{a}_{-k+q}^{+i r}\right):\right\rangle$, имеющие порядок $\nu^{4}$, и члены более высокого порядка в рассматриваемом приближении отбрасываются.

Переход от (2.18) с помощью (3.3) к тождеству (3.4) позволяет, отбрасывая согласно (3.5) члены порядка $\nu^{4}$, получить замкнутое уравнение, содержащее величины порядка $\nu^{2}$. Умножая $(3.4)$ на $\widetilde{G}_{q, E}(p, k) \eta_{q}^{-1}(p, k)$, для $G_{q, E}\left(p, k \mid p^{\prime}, k^{\prime}\right)$ получаем окончательное уравнение

$$
\begin{aligned}
G_{q, E}(p, k \mid & \left.p^{\prime}, k^{\prime}\right)=\widetilde{G}_{q, E}(p, k) \eta_{q}^{-1}(p, k) \eta_{q}\left(p, k \mid p^{\prime}, k^{\prime}\right)+\widetilde{G}_{q, E}(p, k) \eta_{q}^{-1}(p, k) \times \\
& \times\left\{V_{q}(p, k \mid \bar{p}, \bar{k})+\widetilde{U}_{q, E}\left(p, k \mid \bar{p}^{\prime}, \bar{k}^{\prime}\right) \eta_{q}^{-1}\left(\bar{p}^{\prime}, \bar{k}^{\prime} \mid \bar{p}, \bar{k}\right)\right\} G_{q E}\left(\bar{p}, \bar{k} \mid p^{\prime}, k^{\prime}\right),
\end{aligned}
$$


где в обозначении $\widetilde{U}$ учтена разница между $U$ и $U^{(1)}$ в $(3.4)$ и часть порядка $\nu^{2}$ в (3.5).

Расцепление, приводящее к (3.6), является по сравнению с (2.20) оптимальным. Оно приводит к ускоренной сходимости (в уравнении (3.6) отбрасываются члены порядка $\nu^{4}$, в то время как в уравнении $(2.20)$ - члены порядка $\nu^{2}$ ). Благодаря учету членов порядка $\nu^{2}$ (стационарных и зависящих от $E$ ) функция Грина свободных пропагаторов, пропорциональная $\left(E-\varepsilon_{q}(p, k)\right)^{-1}$, в $(2.20)$ заменяется в (3.6) на функцию $\widetilde{G}_{q, E}(p, k)$, выражающуюся через произведения трех одночастичных функций (3.1), что характерно для приближения взаимодействующих мод.

В результате оптимального расцепления мы пришли к самосогласованной системе нелинейных уравнений (2.1), (2.3) и (3.6) для функций $G_{q, E}=\left\langle\left\langle a_{q} \mid a_{q}^{+}\right\rangle\right\rangle_{E}$, $\left\langle\left\langle a_{p}^{+} a_{p+k} a_{-k+q} \mid a_{q}^{+}\right\rangle\right\rangle_{E}$ и $G_{q, E}\left(p, k \mid p^{\prime}, k^{\prime}\right)=\left\langle\left\langle a_{p}^{+} a_{p+k} a_{-k+q} \mid a_{-k^{\prime}+q}^{+} a_{p^{\prime}+k^{\prime}}^{+} a_{p^{\prime}}\right\rangle\right\rangle_{1, E}$ и связанных с ними спектральными представлениями стационарных функций.

Перейдя с помощью спектральных представлений [3] к корреляционным функциям, будем иметь соотношения, связывающие все входящие в уравнения величины $\left\langle a_{q}^{+} a_{q}\right\rangle,\left\langle: a_{q}^{+} a_{p}^{+} a_{p+k} a_{-k+q}:\right\rangle$ и $\left\langle: a_{p}^{+} a_{-k^{\prime}+q}^{+} a_{p^{\prime}+k^{\prime}}^{+} a_{p^{\prime}} a_{p+k} a_{-k+q}:\right\rangle$. При этом единственными определяющими систему частиц параметрами являются плотность и фурье-образ потенциала парного взаимодействия. Остальные функции входят как самосогласованные решения нелинейных уравнений.

\section{4. ЗАКЛЮЧЕНИЕ}

Предложенное в работе приближение, осуществляемое путем оптимального обрыва цепочки уравнений для одночастичной функции (2.1), (2.2), применимо также и в других задачах статистической механики. Ранее оно кратко рассматривалось в [5] при исследовании уравнений молекулярной гидродинамики. Характерной особенностью метода является построение вспомогательного уравнения, которое позволяет заменить появляющиеся в процессе вычислений свободные пропагаторы на точные одночастичные корреляционные функции. Другими словами, мы приходим в данном случае к приближению взаимодействующих мод, которое широко применялось в рамках метода Мори (см., например, работы [6]), но только интуитивно, без должного теоретического обоснования. Изложенный в настоящей работе подход может помочь в достижении этой цели.

\section{Список литературы}

[1] Ю.А. Церковников, ТМФ, 129:3 (2001), 432.

[2] Н. Н. Боголюбов, Д. В. Ширков, Квантовые поля, Наука, М., 1980.

[3] Д. Н. Зубарев, УФН, 71:1 (1960), 71.

[4] Д. Н. Зубарев, Ю. А. Церковников, Тр. МИАН, 175 (1986), 134.

[5] Ю. А. Церковников, Тр. МИАН, 228 (2000), 286.

[6] W.S. Götze, M. Lücke, Phys. Rev. A, 11 (1975), 2173; S. Yip, Ann. Rev. Phys. Chem., 30 (1979), 547. 